\title{
Pacific
}

Journal of

Mathematics

\section{ARITHMETICITY OF \\ COMPLEX HYPERBOLIC TRIANGLE GROUPS}

MATTHEW StOver 


\title{
ARITHMETICITY OF COMPLEX HYPERBOLIC TRIANGLE GROUPS
}

\author{
MATTHEW StOVER
}

\begin{abstract}
Complex hyperbolic triangle groups, originally studied by Mostow in building the first nonarithmetic lattices in $\operatorname{PU}(2,1)$, are a natural generalization of the classical triangle groups. A theorem of Takeuchi states that there are only finitely many Fuchsian triangle groups that determine an arithmetic lattice in $\mathrm{PSL}_{2}(\mathbb{R})$, so triangle groups are generically nonarithmetic. We prove similar finiteness theorems for complex hyperbolic triangle groups that determine an arithmetic lattice in $\operatorname{PU}(2,1)$.
\end{abstract}

\section{Introduction}

In a seminal paper [1980], Mostow constructed lattices in $\mathrm{PU}(2,1)$ generated by three complex reflections. He not only gave a new geometric method for building lattices acting on the complex hyperbolic plane, but gave the first examples of nonarithmetic lattices in $\mathrm{PU}(2,1)$. Complex reflection groups are a generalization of groups generated by reflections through hyperplanes in constant curvature spaces, and Mostow's groups are a natural extension to the complex hyperbolic plane of the classical triangle groups. They are often called complex hyperbolic triangle groups. We introduce these groups in Section 2. See also [Goldman and Parker 1992; Schwartz 2002], which, along with [Mostow 1980], inspired much of the recent surge of activity surrounding these groups.

Around the same time, Takeuchi [1977] classified the Fuchsian triangle groups that determine arithmetic lattices in $\mathrm{PSL}_{2}(\mathbb{R})$. In particular, he proved that there are finitely many and gave a complete list. Since there are infinitely many triangle groups acting on the hyperbolic plane discretely with finite covolume, triangle groups are generically nonarithmetic. The purpose of this paper is to give analogous finiteness results for complex hyperbolic triangle groups that determine an arithmetic lattice in $\mathrm{PU}(2,1)$.

A particular difficulty with complex hyperbolic triangle groups is that the complex triangle is not uniquely determined by its angles. One must also consider the

Partially supported by NSF RTG grant DMS 0602191.

MSC2010: 11F06, 20H10, 22E40.

Keywords: complex hyperbolic geometry, arithmetic lattices, complex hyperbolic triangle groups. 
so-called angular invariant $\psi \in[0,2 \pi)$. See Section 2. In particular, there is a 1-dimensional deformation space of complex triangles with fixed triple of angles. The typical assumption is that $\psi$ is a rational multiple of $\pi$, in which case the angular invariant is called rational. We call it irrational otherwise.

When a complex hyperbolic triangle group is also an arithmetic lattice, we will call it an arithmetic complex hyperbolic triangle group. Note that this immediately implies discreteness. Our first result is for nonuniform arithmetic complex hyperbolic triangle groups. We prove the following in Section 6.

Theorem 1.1. There are finitely many nonuniform arithmetic complex hyperbolic triangle groups with rational angular invariant. If $\Gamma$ is a nonuniform arithmetic complex hyperbolic triangle group with irrational angular invariant $\psi$, then $e^{i \psi}$ is contained in a biquadratic extension of $\mathbb{Q}$.

We emphasize that complex reflection groups are allowed to have generators of arbitrary finite order. A usual assumption is that all generators have the same order, a restriction that we avoid. See Theorem 6.1 for a more precise formulation of Theorem 1.1. Proving that a candidate is indeed a lattice is remarkably difficult, as evidenced in [Mostow 1980; Deraux et al. 2011], so we do not give a definitive list. One consequence of the proof (see Theorem 1.5(1) below) is the following.

Corollary 1.2. Suppose that $\Gamma$ is a nonuniform lattice in $\mathrm{U}(2,1)$. If $\Gamma$ contains a complex reflection of order 5 or at least 7 , then $\Gamma$ is nonarithmetic.

In the cocompact setting, the arithmetic is much more complicated. Arithmetic subgroups of $U(2,1)$ come in two types, defined in Section 3, often called first and second. In Section 4 we prove the following auxiliary result, generalizing a well-known fact for hyperbolic reflection groups.

Theorem 1.3. Let $\Gamma<\mathrm{U}(2,1)$ be a lattice containing a complex reflection. Then $\Gamma$ contains a Fuchsian subgroup stabilizing the wall of the reflection in $\mathbf{H}_{\mathbb{C}}^{2}$

We also give a generalization to higher-dimensional complex reflection groups. Theorem 1.3 leads to the following, which we also prove in Section 4.

Theorem 1.4. Let $\Gamma<\mathrm{U}(2,1)$ be a lattice, and suppose that $\Gamma$ is commensurable with a lattice $\Lambda$ containing a complex reflection. Then $\Gamma$ is either arithmetic of first type or nonarithmetic.

In particular, when considering a complex reflection group as a candidate for a nonarithmetic lattice, one must only show that it is not of the first type. Fortunately, this is the case where the arithmetic is simplest to understand.

The effect of the angular invariant is a particular sticking point in generalizing Takeuchi's methods. In Section 5, the technical heart of the paper, we study the interdependence between the geometric invariants of the triangle and the arithmetic 
of the lattice. We collect the most useful of these facts as the following. See $\S \S 2-3$ for our notation.

Theorem 1.5. Suppose that $\Gamma$ is an arithmetic complex hyperbolic triangle group. Suppose that for $j=1,2,3$ the generators have reflection factors $\eta_{j}$, the complex angles of the triangle are $\theta_{j}$, and that the angular invariant is $\psi$. Let $E$ be the totally imaginary quadratic extension of the totally real field $F$ that defines $\Gamma$ as an arithmetic lattice. Then:

(1) $\eta_{j} \in$ E for all $j$;

(2) $\cos ^{2} \theta_{j} \in F$ for all $j$;

(3) $e^{2 i \psi} \in E$ and $\cos ^{2} \psi \in F$;

(4) If $\theta_{j} \leq \pi / 3$ for all $j$, then

$$
\cos ^{2} \psi \in \mathbb{Q}\left(\cos ^{2} \theta_{1}, \cos ^{2} \theta_{2}, \cos ^{2} \theta_{3}, \cos \theta_{1} \cos \theta_{2} \cos \theta_{3}\right) ;
$$

(5) $E \subseteq \mathbb{Q}\left(\cos ^{2} \theta_{1}, \cos ^{2} \theta_{2}, \cos ^{2} \theta_{3}, e^{i \psi} \cos \theta_{1} \cos \theta_{2} \cos \theta_{3}\right)$;

(6) If $\psi$ is rational, then $E$ is a subfield of a cyclotomic field.

In Section 6, we use the results from Section 5 to prove finiteness results for cocompact arithmetic complex hyperbolic triangle groups with rational angular invariant. We also give restrictions for irrational angular invariants, though it is unknown whether such a lattice exists. When the complex triangle is a right triangle, we prove the following.

Theorem 1.6. Suppose that $\Gamma$ is an arithmetic complex hyperbolic triangle group for which the associated complex triangle is a right triangle. Then the angles of the triangle are the angles of an arithmetic Fuchsian triangle group. There are finitely many such $\Gamma$ with rational angular invariant.

Finally, we consider equilateral triangles at the end of Section 6. This is the case which has received the most attention, in particular from Mostow [1980] and, in the ideal case, by Goldman and Parker [1992] and Schwartz [2002]. See also [Deraux 2006]. Here we cannot explicitly bound orders of generators, angles, or angular invariants because our proof relies on asymptotic number theory for which we do not know precise constants. Nevertheless, we obtain finiteness in the situation that has received the greatest amount of attention since Mostow's original paper. See [Parker 2008; Parker and Paupert 2009; Paupert 2010; Deraux et al. 2011] and references therein for more recent examples of lattices and restrictions on discreteness.

Theorem 1.7. There are finitely many arithmetic complex hyperbolic equilateral triangle groups with rational angular invariant. 


\section{Complex hyperbolic triangle groups}

We assume some basic knowledge of complex hyperbolic geometry, e.g., the first three chapters of [Goldman 1999]. Let $V$ be a three-dimensional complex vector space, equipped with a hermitian form $h$ of signature $(2,1)$. Complex hyperbolic space $\mathbf{H}_{\mathbb{C}}^{2}$ is the space of $h$-negative lines in $V$. The metric on $\mathbf{H}_{\mathbb{C}}^{2}$ is defined via $h$ as in [Goldman 1999, Chapter 3], and the action of $\mathbf{U}(2,1)$ on $\mathbf{H}_{\mathbb{C}}^{2}$ by isometries descends from its action on $V$ and factors through projection onto $\mathrm{PU}(2,1)$. Its ideal boundary $\partial \mathbf{H}_{\mathbb{C}}^{2}$ is the space of $h$-isotropic lines, and we set $\overline{\mathbf{H}}_{\mathbb{C}}^{2}=\mathbf{H}_{\mathbb{C}}^{2} \cup \partial \mathbf{H}_{\mathbb{C}}^{2}$.

A complex reflection is a diagonalizable linear map $R: V \rightarrow V$ with one eigenvalue of multiplicity 2 (or, more generally, multiplicity $n-1$ when $\operatorname{dim}(V)=n$ ). We assume that $R$ has finite order, so the third eigenvalue is a root of unity $\eta$. We call $\eta$ the reflection factor of $R$. Decompose $V=V_{1} \oplus V_{\eta}$ into the 1- and $\eta$-eigenspaces, and choose $v_{\eta} \in V$ so that $V_{\eta}=\operatorname{Span}_{\mathbb{C}}\left\{v_{\eta}\right\}$. We begin with an elementary lemma that will be of use later, keeping in mind that every complex reflection has 1 as an eigenvalue.

Lemma 2.1. Let $A \in \mathrm{GL}_{n}(\mathbb{C})$ be a diagonalizable linear transformation. Let $E \subseteq \mathbb{C}$ be a subfield, and suppose that $E^{n}$ has a basis consisting of eigenvectors for $A$. Furthermore, suppose that $A$ has at least one eigenvalue in $E$ and that there exists $x \in \mathbb{C}^{\times}$so that $x A \in \mathrm{GL}_{n}(E)$. Then all eigenvalues of $A$ are in $E$.

Proof. Let $v_{1}, \ldots, v_{n} \in E^{n}$ be a basis of eigenvectors for $A$, and let $\lambda_{j}$ be the eigenvalue associated with $v_{j}, 1 \leq j \leq n$. Without loss of generality, $\lambda_{1} \in E$. Then $x A$ also has eigenvectors $v_{1}, \ldots, v_{n}$, and $x A v_{j}=x \lambda_{j} v_{j} \in E^{n}$ for all $j$, since $x A \in \mathrm{GL}_{n}(E)$. Then $x \lambda_{j} \in E, 1 \leq j \leq n$. Since $\lambda_{1} \in E$, it follows that $x \in E$, which implies that $\lambda_{j} \in E$ for all $j$.

Assume that $R \in \mathrm{U}(2,1)$. Then the fixed point set of $R$ acting on $\mathbf{H}_{\mathbb{C}}^{2}$ is the subset of $h$-negative lines in $V_{1}$. This is a totally geodesic holomorphic embedding of the hyperbolic plane if and only if $V_{\eta}$ is an $h$-positive line. These subspaces are called complex hyperbolic lines. Following [Goldman 1999, §3.1], we call $v_{\eta}$ a polar vector for $R$.

When $V_{\eta}$ is $h$-negative, the fixed set of $R$ on $\mathbf{H}_{\mathbb{C}}^{2}$ is a point, and $R$ is sometimes called a reflection through that point. The complex reflections in this paper will always be through complex hyperbolic lines. That is, the $\eta$-eigenspace will always be an $h$-positive line.

Let $W$ be the complex hyperbolic line in $\mathbf{H}_{\mathbb{C}}^{2}$ fixed by $R$. We call this the wall of $R$. If $v_{\eta}$ is a polar vector, then $R$ is the linear transformation

$$
z \mapsto z+(\eta-1) \frac{h\left(z, v_{\eta}\right)}{h\left(v_{\eta}, v_{\eta}\right)} v_{\eta}
$$


We refrain from normalizing the polar vector to have $h$-norm one, since we will often choose a polar vector with coordinates in a subfield $E$ of $\mathbb{C}$, and $E^{3} \subset V$ might not contain an $h$-norm one representative for the given line of polar vectors.

Now, consider three complex reflections $R_{1}, R_{2}, R_{3} \in \mathrm{U}(2,1)$ with respective distinct walls $W_{1}, W_{2}, W_{3}$ in $\mathbf{H}_{\mathbb{C}}^{2}$. If $v_{j}$ is a polar vector for $R_{j}$, then $W_{j}$ and $W_{j+1}$ (with cyclic indices) meet in $\mathbf{H}_{\mathbb{C}}^{2}$ if and only if

$$
h\left(W_{j}, W_{j+1}\right)=\frac{\left|h\left(v_{j}, v_{j+1}\right)\right|^{2}}{h\left(v_{j}, v_{j}\right) h\left(v_{j+1}, v_{j+1}\right)}<1 .
$$

The two walls meet at a point $z_{j}$ stabilized by the subgroup of $\mathrm{U}(2,1)$ generated by $R_{j}$ and $R_{j+1}$. The complex angle $\theta_{j}$ between $W_{j}$ and $W_{j+1}$, the minimum angle between the two walls, satisfies $\cos ^{2} \theta_{j}=h\left(W_{j}, W_{j+1}\right)$.

The walls $W_{j}$ and $W_{j+1}$ meet at a point $p_{j}$ in $\partial \mathbf{H}_{\mathbb{C}}^{2}$ if and only if

$$
\frac{\left|h\left(v_{j}, v_{j+1}\right)\right|^{2}}{h\left(v_{j}, v_{j}\right) h\left(v_{j+1}, v_{j+1}\right)}=1,
$$

so we say that the complex angle is zero. The group generated by $R_{j}$ and $R_{j+1}$ fixes $p_{j}$, so it is contained in a parabolic subgroup of $\mathrm{U}(2,1)$. See [Goldman 1999, \$3.3.2].

Let $\left\{R_{j}\right\}$ be reflections through walls $\left\{W_{j}\right\}, j=1,2,3$. When the pairwise intersections of the walls are nontrivial in $\overline{\mathbf{H}}_{\mathbb{C}}^{2}$, they determine a complex triangle in $\mathbf{H}_{\mathbb{C}}^{2}$, possibly with ideal vertices. The subgroup $\triangle\left(R_{1}, R_{2}, R_{3}\right)$ of $\mathrm{U}(2,1)$ generated by the $R_{j}$ s is called a complex hyperbolic triangle group.

A complex hyperbolic triangle group is sometimes defined as one with order two generators, and groups with higher order generators are called generalized triangle groups. We avoid this distinction and do not make the usual assumption that all generators have the same order.

Unlike Fuchsian triangle groups, the complex angles $\left\{\theta_{1}, \theta_{2}, \theta_{3}\right\}$ do not suffice to determine $\triangle\left(R_{1}, R_{2}, R_{3}\right)$ up to $\operatorname{Isom}\left(\mathbf{H}_{\mathbb{C}}^{2}\right)$-equivalence. We also need to consider Cartan's angular invariant

$$
\psi=\arg \left(h\left(v_{1}, v_{2}\right) h\left(v_{2}, v_{3}\right) h\left(v_{3}, v_{1}\right)\right) .
$$

A complex triangle is uniquely determined up to complex hyperbolic isometry by the complex angles between the walls and the angular invariant. See [Brehm 1990] and [Pratoussevitch 2005, Proposition 1]. Up to the action of complex conjugation on $\mathbf{H}_{\mathbb{C}}^{2}$, we can assume $\psi \in[0, \pi]$.

We call the angular invariant rational if $\psi=s \pi / t$ for some (relatively prime) $s, t \in \mathbb{Z}$. In other words, the angular invariant is rational if and only if $e^{i \psi}$ is a root of unity. 
Let $\triangle\left(R_{1}, R_{2}, R_{3}\right)$ be a complex hyperbolic triangle group in $\mathrm{U}(2,1)$ with reflection factors $\eta_{j}$, complex angles $\theta_{j}$, polar vectors $v_{j}, j=1,2,3$, and angular invariant $\psi$. Suppose that $\left\{v_{1}, v_{2}, v_{3}\right\}$ is a basis for $V$. Then $\triangle\left(R_{1}, R_{2}, R_{3}\right)$ preserves the hermitian form

$$
h_{\triangle\left(R_{1}, R_{2}, R_{3}\right)}=\left(\begin{array}{ccc}
1 & e^{i \psi} \cos \theta_{1} & e^{i \psi} \cos \theta_{3} \\
e^{-i \psi} \cos \theta_{1} & 1 & e^{i \psi} \cos \theta_{2} \\
e^{-i \psi} \cos \theta_{3} & e^{-i \psi} \cos \theta_{2} & 1
\end{array}\right) .
$$

We denote this by $h_{\triangle}$ when the generators are clear.

\section{Arithmetic subgroups of $U(2,1)$}

Let $F$ be a totally real number field, $E$ a totally imaginary quadratic extension, and D a central simple $E$-algebra of degree $d$. Let $\tau: \mathscr{D} \rightarrow \mathscr{D}$ be an involution, that is, an antiautomorphism of order two. Then $\tau$ is of second kind if $\left.\tau\right|_{E}$ is the Galois involution of $E / F$. There are two cases of interest.

(1) If $\mathscr{D}=E$ (i.e., $d=1$ ), then $\tau$ is the Galois involution.

(2) If $d=3$, then $\mathscr{D}$ is a cubic division algebra with center $E$.

See [Knus et al. 1998] for more on algebras with involution.

For $d$ as above, let $r=3 / d$. A form $h: \mathscr{D}^{r} \rightarrow \mathscr{D}$ is called hermitian or $\tau$ hermitian if it satisfies the usual definition of a hermitian form with $\tau$ in place of complex conjugation. If $d=1$, then $h$ is a hermitian form on $E^{3}$ as usual. If $d=3$, then there exists an element $x \in \mathscr{D}^{*}$ such that $\tau(x)=x$ and $h\left(y_{1}, y_{2}\right)=\tau\left(y_{1}\right) x y_{2}$ for all $y_{1}, y_{2} \in \mathscr{D}$.

This determines an algebraic group $\mathscr{G}$, the group of elements in $\mathrm{GL}_{r}(\mathscr{D})$ preserving $h$. For every embedding $\iota: F \rightarrow \mathbb{R}$, we obtain an embedding of $\varphi$ into the real Lie group $\mathrm{U}(\iota(h))$. Let $\bar{G}$ be the associated projective unitary group.

If $\mathcal{O}$ is a order in $\mathscr{D}^{r}$, then the subgroup $\Gamma_{\mathscr{O}}$ of $\mathrm{GL}_{r}(\mathcal{O})$ preserving $h$ embeds as a discrete subgroup of

$$
\mathscr{G}(\mathbb{R})=\prod_{\iota: F \rightarrow \mathbb{R}} \mathrm{U}(\iota(h))
$$

If $\bar{\Gamma}_{\mathscr{O}}$ is the image of $\Gamma_{\mathscr{O}}$ in $\overline{\mathscr{G}}$, then $\bar{\Gamma}_{\mathscr{O}}$ is a discrete subgroup of the associated product of projective unitary groups.

The projection of $\Gamma_{\mathscr{O}}$ onto any factor of $\mathscr{G}(\mathbb{R})$ is discrete if and only if the kernel of the projection of $\mathscr{G}(\mathbb{R})$ onto that factor is compact. Therefore, we obtain a discrete subgroup of $\mathrm{U}(2,1)$ if and only if $\mathrm{U}(\iota(h))$ is noncompact for exactly one real embedding of $F$.

Then $\bar{\Gamma}_{0}$ is a lattice in PU $(2,1)$ by the well-known theorem of Borel and HarishChandra. An arithmetic lattice in $\operatorname{PU}(2,1)$ is any lattice $\Gamma<\operatorname{PU}(2,1)$ which is commensurable with $\bar{\Gamma}_{\mathscr{O}}$ for some $\mathscr{G}$ as above and an order $\mathscr{O}$ in $\mathscr{D}$. 
Since arithmeticity only requires commensurability with $\Gamma_{\mathscr{O}}$, studying an arbitrary $\Gamma$ in the commensurability class of $\Gamma_{\mathscr{C}}$ requires great care. The image of any element $\gamma \in \Gamma$ in $\mathrm{PU}(2,1)$ does, however, have a representative in $\mathrm{GL}_{3}(E)$, that is, there exists $x \in \mathbb{C}^{\times}$so $x \gamma \in \mathrm{GL}_{3}(E)$. This follows from the fact, due to Vinberg [1971], that $\Gamma$ is $F$-defined over the adjoint form $\overline{\mathscr{G}}$, i.e.,

$$
\mathbb{Q}(\operatorname{Tr} \operatorname{Ad} \Gamma)=F .
$$

This important fact also follows from [Platonov and Rapinchuk 1994, Proposition 4.2].

\section{Proofs of Theorems 1.3 and 1.4}

We require some elementary results from the theory of discrete subgroups of Lie groups. The primary reference is [Raghunathan 1972]. Let $G$ be a second countable, locally compact group and $\Gamma<G$ a lattice. Recall that $G / \Gamma$ carries a finite $G$-invariant measure and $\Gamma$ is uniform in $G$ if $G / \Gamma$ is compact. For a subgroup $H<G$, we let $Z_{G}(H)$ denote the centralizer of $H$ in $G$. We need the following two results.

Lemma 4.1 [Raghunathan 1972, Lemma 1.14]. Let $G$ be a second countable locally compact group, $\Gamma<G$ a lattice, $\Delta \subset \Gamma$ a finite subset, and $Z_{G}(\Delta)$ the centralizer of $\Delta$ in $G$. Then, $Z_{G}(\Delta) \Gamma$ is closed in $G$.

Theorem 4.2 [Raghunathan 1972, Theorem 1.13]. Let $G$ be a second countable locally compact group, $\Gamma<G$ be a uniform lattice, and $H<G$ be a closed subgroup. Then $H \Gamma$ is closed in $G$ if and only if $H \cap \Gamma$ is a lattice in $H$.

Proof of Theorem 1.3. Assume that $\Gamma$ is a cocompact arithmetic lattice in $\mathrm{U}(2,1)$ containing a complex reflection and that $\Delta$ is the subgroup of $\Gamma$ generated by this reflection. The centralizer of $\Delta$ in $\mathrm{U}(2,1)$ is isomorphic to the extension of $\mathrm{U}(1,1)$ by the center of $U(2,1)$, and is the stabilizer in $U(2,1)$ of the wall of the reflection that generates $\Delta$. It follows from Lemma 4.1 and Theorem 4.2 that $\Gamma \cap \mathrm{U}(1,1)$ is a lattice. Since any sublattice of an arithmetic lattice is arithmetic, $\Gamma$ contains a totally geodesic arithmetic Fuchsian subgroup.

Proof of Theorem 1.4. A totally geodesic arithmetic Fuchsian group comes from a subalgebra of $\mathscr{D}^{r}$, with notation as in Section 3. When $\Gamma$ is of second type, $\mathscr{D}$ is a cubic division algebra. The totally geodesic Fuchsian group would correspond to a quaternion subalgebra of $\mathscr{D}$, which is impossible. When $\Gamma$ is of first type, this quaternion subalgebra corresponds to rank 2 subspaces of $E^{3}$ on which $h$ has signature $(1,1)$. Therefore, $\Gamma$ contains complex reflections if and only if $\Gamma$ is of first type. 
Remark. One can also prove Theorem 1.4 using the structure of unit groups of division algebras.

We now briefly describe how these results generalize to reflections acting on higher-dimensional complex hyperbolic spaces. If $\Gamma<\mathrm{U}(n, 1)$ is a lattice, an element $R \in \Gamma$ is a codimension s reflection if it stabilizes a totally geodesic embedded $\mathbf{H}_{\mathbb{C}}^{n-s}$ and acts by an element of the unitary group of the normal bundle to the wall. If $\Gamma$ is arithmetic, the associated algebraic group is constructed via a hermitian form on $\mathscr{D}^{r}$, where $\mathscr{D}$ is a division algebra of degree $d$ with involution of the second kind over a totally imaginary field $E$, and where $r d=n+1$.

Theorem 4.3. Suppose $\Gamma<\mathrm{U}(n, 1)$ is a cocompact arithmetic lattice with associated algebraic group coming from a hermitian form on $\mathscr{D}^{r}$, where $\mathscr{D}$ is a central simple algebra with involution of the second kind. If $\Gamma$ contains a codimension $s$ reflection, then $\Gamma$ contains a cocompact lattice in $\mathrm{U}(n-s, 1)$. Also, $n-s+1=\ell d$ for some $1<\ell \leq r$ and the associated algebraic subgroup comes from a hermitian form on $\mathscr{D}^{\ell}$.

Corollary 4.4. Let $\Gamma<\mathrm{U}(n, 1)$ be an arithmetic lattice generated by complex reflections through totally geodesic walls isometric to $\mathbf{H}_{\mathbb{C}}^{n-1}$. Then $\Gamma$ is of so-called first type, i.e., the associated algebraic group is the automorphism group of a hermitian form on $E^{n+1}$, where $E$ is some totally imaginary quadratic extension of a totally real field.

\section{Arithmetic data for complex hyperbolic triangle groups}

In this section, we relate the geometric invariants of a complex triangle to the arithmetic invariants of the complex reflection group. It is the technical heart of the paper.

Let $\Gamma=\triangle\left(R_{1}, R_{2}, R_{3}\right)$ be a complex hyperbolic triangle group with reflection factors $\eta_{j}$, complex angles $\theta_{j}$, and angular invariant $\psi$. Assume that $\Gamma$ is an arithmetic lattice in $\mathrm{U}(2,1)$. By Theorem $1.4, \Gamma$ is of first type, so there is an associated hermitian form $h$ over a totally imaginary field $E$. Let $F$ be the totally real quadratic subfield of $E$.

Lemma 5.1. We can choose polar vectors $v_{j}$ for the reflection $R_{j}$ so that $v_{j} \in E^{3}$.

Proof. Associated with each reflection is an arithmetic Fuchsian subgroup of $\Gamma$. When $\Gamma$ is a uniform lattice, this follows from Theorem 1.3. For the nonuniform case, see [Holzapfel 1998, Chapter 5]. Arithmetic Fuchsian subgroups stabilizing a complex hyperbolic line come from the $h$-orthogonal complement of an $h$-positive line in $E^{3}$. (To be more precise, this line is $h$-positive over the unique real embedding of $F$ at which $h$ is indefinite.) Any vector in $E^{3}$ representing this line is a polar vector for $R_{j}$. 
This leads us to the following important fact.

Lemma 5.2. Each reflection factor $\eta_{j}$ is contained in $E$.

Proof. It follows from Proposition 4.2 in [Platonov and Rapinchuk 1994] that there exists an $x_{j} \in \mathbb{C}$ so that $x_{j} R_{j} \in \mathrm{GL}_{3}(E)$ (see the end of Section 3 above). By Lemma 5.1, and because the $h$-orthogonal complement to a polar vector evidently has an $E$-basis, $E^{3}$ has a basis of eigenvectors for $R_{j}$. The lemma follows from Lemma 2.1.

Now we turn to the complex angles and the angular invariant.

Lemma 5.3. For each $j, \cos ^{2} \theta_{j} \in F$ and $e^{2 i \psi} \in E$.

Proof. Choose polar vectors $v_{j} \in E^{3}$. The terms in Equations (2) and (3) resulting from these choices of polar vectors are all contained in $E$. Hence $\cos ^{2} \theta_{j} \in F$. One can also prove this using $\operatorname{Tr} \operatorname{Ad}\left(R_{1} R_{2}\right)$ and Lemma 5.2.

Similarly, consider

$$
\delta=h\left(v_{1}, v_{2}\right) h\left(v_{2}, v_{3}\right) h\left(v_{3}, v_{1}\right)=r e^{i \psi} \in E
$$

from (4). Note that $e^{i \psi} \in E$ if and only if $r \in E$. Either way, when $\delta \neq 0$, we have $\delta / \bar{\delta}=e^{2 i \psi} \in E$. This completes the proof.

Combining the above, we see that

$$
\mathbb{Q}\left(\eta_{1}, \eta_{2}, \eta_{3}, \cos ^{2} \theta_{1}, \cos ^{2} \theta_{2}, \cos ^{2} \theta_{3}, e^{2 i \psi}\right) \subseteq E .
$$

We can also bound $E$ from above using the fact that $E \subseteq \mathbb{Q}(\operatorname{Tr} \Gamma)$. Using wellknown computations of traces for products of reflections (e.g., [Mostow 1980, §4] or [Pratoussevitch 2005]), we have

$$
\mathbb{Q}(\operatorname{Tr} \Gamma)=\mathbb{Q}\left(\eta_{1}, \eta_{2}, \eta_{3}, \cos ^{2} \theta_{1}, \cos ^{2} \theta_{2}, \cos ^{2} \theta_{3}, e^{i \psi} \cos \theta_{1} \cos \theta_{2} \cos \theta_{3}\right) .
$$

Similarly,

$\mathbb{Q}\left(\operatorname{Re} \eta_{1}, \operatorname{Re} \eta_{2}, \operatorname{Re} \eta_{3}, \cos ^{2} \theta_{1}, \cos ^{2} \theta_{2}, \cos ^{2} \theta_{3}, \cos ^{2} \psi\right) \subseteq F$

$\subseteq \mathbb{Q}\left(\operatorname{Re} \eta_{1}, \operatorname{Re} \eta_{2}, \operatorname{Re} \eta_{3}, \cos ^{2} \theta_{1}, \cos ^{2} \theta_{2}, \cos ^{2} \theta_{3}, \cos \psi \cos \theta_{1} \cos \theta_{2} \cos \theta_{3}\right)$.

This gives the following.

Corollary 5.4. Let $\Gamma$ be a complex hyperbolic triangle group and an arithmetic lattice in $\mathrm{U}(2,1)$. If the angular invariant of the triangle associated with $\Gamma$ is rational, then the fields that define $\Gamma$ as an arithmetic lattice are subfields of a cyclotomic field.

Let $h_{\triangle}$ be as in (5) and consider $h_{\triangle}$ as a hermitian form on the extension

$$
E_{\Delta}=\mathbb{Q}\left(\eta_{1}, \eta_{2}, \eta_{3}, \cos \theta_{1}, \cos \theta_{2}, \cos \theta_{3}, e^{i \psi}\right),
$$


of $E$. It follows from [Mostow 1980, §2] that $h$ and $h_{\triangle}$ are equivalent over $E_{\triangle}$. Consequently, $h_{\triangle}$ is indefinite over exactly one complex conjugate pair of places of $E$. This implies that there are precisely $\left[E_{\triangle}: E\right]$ conjugate pairs of places of $E_{\triangle}$ over which $h_{\triangle}$ is indefinite.

Let $H$ be a hermitian form in 3 variables over the complex numbers for which there is a vector with positive $H$-norm. Then $H$ is indefinite if and only if det $H<0$. Since any polar vector has positive $h_{\triangle}$-norm by definition, we have the following.

Proposition 5.5. There are exactly $\left[E_{\triangle}: E\right]$ complex conjugate pairs of Galois automorphisms $\tau$ of $E_{\triangle} \subset \mathbb{C}$ under which $\tau\left(\operatorname{det} h_{\triangle}\right)$ is negative. All such automorphisms act trivially on $E$.

This has the following consequence for the relationship between the geometry of the triangle and the arithmetic of the lattice.

Corollary 5.6. If $\Gamma$ is a complex hyperbolic triangle group and an arithmetic lattice, then the reflection factors of $\Gamma$ are restricted by the geometry of the triangle. In particular,

$$
E_{\triangle}=\mathbb{Q}\left(\cos \theta_{1}, \cos \theta_{2}, \cos \theta_{3}, e^{i \psi}\right) .
$$

Proof. Since det $h_{\triangle}$ is independent of the reflection factors, for each Galois automorphism of

$$
E_{\triangle} / \mathbb{Q}\left(\cos \theta_{1}, \cos \theta_{2}, \cos \theta_{3}, e^{i \psi}\right)
$$

we obtain a new complex conjugate pair of embeddings of $E_{\triangle}$ into $\mathbb{C}$ such that $\operatorname{det} h_{\triangle}$ is negative. Any such automorphism necessarily acts nontrivially on some reflection factor $\eta_{j}$. These embeddings of $E_{\Delta}$ lie over different places of $E$ by Lemma 5.2. This contradicts Proposition 5.5.

We also obtain the following dependence between the angular invariant and the angles of the triangle.

Proposition 5.7. If $\Gamma$ is a complex hyperbolic triangle group and an arithmetic lattice. If $\Gamma$ has rational angular invariant and $\theta_{j} \leq \pi / 3$ for $j=1,2,3$, then

$$
\cos ^{2} \psi \in F^{\prime}=\mathbb{Q}\left(\cos ^{2} \theta_{1}, \cos ^{2} \theta_{2}, \cos ^{2} \theta_{3}, \cos \theta_{1} \cos \theta_{2} \cos \theta_{3}\right) .
$$

Proof. If $\psi$ is rational, then $E_{\triangle}$ is a subfield of a cyclotomic field $K_{N}=\mathbb{Q}\left(\zeta_{N}\right)$, where $\zeta_{N}$ is a primitive $N$-th root of unity. Therefore the Galois automorphisms of $E_{\triangle}$ are induced by $\zeta_{N} \mapsto \zeta_{N}^{m}$ for some $m$ relatively prime to $N$.

Consider the stabilizer $S$ of $F^{\prime}$ in $\operatorname{Gal}\left(K_{N} / \mathbb{Q}\right)$. It acts on the roots of unity in $E_{\triangle}$ as a group of rotations along with complex conjugation. By definition of $E_{\triangle}$, every nontrivial element of $S$ acts nontrivially on $e^{i \psi}$. In particular, if $\cos ^{2} \psi \notin \mathbb{Q}$ and $S$ contains a rotation through an angle other than an integral multiple of $\pi$, then 
the orbit of $e^{i \psi}$ under $S$ contains two non-complex conjugate points with distinct negative real parts.

Let $\tau$ be any such automorphism of $E_{\triangle}$. Then, since $\tau\left(\cos \theta_{j}\right)=\cos \theta_{j}$ for all $j$ by definition of $S$,

$$
\tau\left(\operatorname{det} h_{\triangle}\right)=1-\sum_{j=1}^{3} \cos ^{2} \theta_{j}+2 \tau(\cos \psi) \prod_{j=1}^{3} \cos \theta_{j} .
$$

Furthermore, $1-\sum \cos ^{2} \theta_{j} \leq 0$ for any triple of angles $\theta_{j}=\pi / r_{j}$ that are the angles of a hyperbolic triangle with each $r_{j} \geq 3$. Since $\tau(\cos \psi)<0$ and $\cos \theta_{j}>0$, it follows that $\tau\left(\operatorname{det} h_{\triangle}\right)<0$. Since $\tau$ acts nontrivially on $e^{2 i \psi} \in E$, this contradicts Proposition 5.5. Therefore, $S$ is generated by complex conjugation and rotation by $\pi$, so $\cos ^{2} \psi \in F^{\prime}$.

Remark. For several of the lattices in [Mostow 1980], $F^{\prime}=F$ (with notation as above) and $\cos \psi \notin F^{\prime}$. Thus Proposition 5.7 is the strongest possible constraint on rational angular invariants.

\section{Finiteness results}

We are now prepared to collect facts from Section 5 to prove Theorem 1.1. A more precise version is the following.

Theorem 6.1. Suppose that $\Gamma$ is a complex hyperbolic triangle group and a nonuniform arithmetic lattice in $\mathrm{U}(2,1)$. Then:

(1) Each generator has order 2, 3, 4, or 6.

(2) Each complex angle $\theta_{j}$ of the triangle comes from the set

$$
\{\pi / 2, \pi / 3, \pi / 4, \pi / 6,0\} \text {. }
$$

(3) If $\psi$ is the angular invariant, then $e^{i \psi}$ lies in a biquadratic extension of $\mathbb{Q}$.

(4) If $\psi$ is rational, then $\psi=s \pi / t$ for

$$
t \in\{2,3,4,6,8,12\} .
$$

Proof. Since $\Gamma$ is a nonuniform arithmetic lattice, the associated field $E$ is imaginary quadratic. For (1), we apply Lemma 5.2 to $E$. For (2) and (3), we apply Lemma 5.3. Then (4) follows from determining those integers $m$ so that $\varphi(m)=2$ or 4 and $e^{2 i \psi}$ is at most quadratic over $\mathbb{Q}$, where $\varphi$ is Euler's totient function.

See [Paupert 2010; Deraux et al. 2011] for the known nonuniform arithmetic complex hyperbolic triangle groups. We now determine the right triangle groups that can determine an arithmetic lattice in $\mathrm{SU}(2,1)$. 
Proof of Theorem 1.6. Suppose that $\Gamma$ is an arithmetic complex hyperbolic triangle group with $\theta_{1}=\pi / 2$. The hermitian form $h_{\triangle}$ associated with the triangle has determinant

$$
1-\cos ^{2} \theta_{2}-\cos ^{2} \theta_{3} .
$$

By Lemma 5.3, this is an element of the totally real field $F$ that defines $\Gamma$ as an arithmetic lattice. Consequently, there is no Galois automorphism of $F$ over $\mathbb{Q}$ under which this expression remains negative.

This is precisely Takeuchi's condition that determines whether or not the triangle in the hyperbolic plane with angles $\pi / 2, \theta_{2}, \theta_{3}$ determines an arithmetic Fuchsian group. The theorem follows from Takeuchi's classification of arithmetic Fuchsian right triangle groups, Lemma 5.3, and Corollary 5.6.

There are 41 such right triangles in $\mathbf{H}^{2}$. We now finish the paper with finiteness for arithmetic complex hyperbolic triangle groups with equilateral complex triangle and rational angular invariant.

Proof of Theorem 1.7. Let $\Gamma$ be an arithmetic complex hyperbolic triangle group with equilateral triangle of angles $\pi / n$ and angular invariant $\psi$. By Proposition 5.7, we can assume that $\psi=s \pi / 12 n$ for some integer $s$. Indeed, $F^{\prime}=\mathbb{Q}(\cos \pi / n)$, and the assertion follows from an easy Galois theory computation.

Then

$$
\operatorname{det} h_{\triangle}=1-3 \cos ^{2}(\pi / n)+2 \cos (s \pi / 12 n) \cos ^{3}(\pi / n),
$$

so we want to find a nontrivial Galois automorphism of $F_{\triangle}$ whose restriction to $F$ is nontrivial and such that the image of (6) under this automorphism is negative. Let $p$ be the smallest rational prime not dividing $12 n$. This determines a nontrivial Galois automorphism $\tau_{p}$ of $F_{\triangle}$ under which

$$
\tau_{p}\left(\operatorname{det} h_{\triangle}\right)=1-3 \cos ^{2}(p \pi / n)+2 \cos (p s \pi / 12 n) \cos ^{3}(p \pi / n) .
$$

It is nontrivial on $F$ by definition. If we show that $\tau_{p}\left(\operatorname{det} h_{\triangle}\right)<0$ for $n$ sufficiently large, this, along with Corollary 5.6, suffices to prove the theorem.

First, notice that the function

$$
D(x, y)=1-3 \cos ^{3}+2 \cos y \cos ^{3} x
$$

is an increasing function of $x \in(0, \pi / 2)$ for any fixed $y$. In our language, this implies that if $y$ is the angular invariant of an equilateral complex triangle in $\mathbf{H}_{\mathbb{C}}^{2}$ with angle $x$, then it remains an angular invariant for a complex triangle with angle $x^{\prime}$ for any $x^{\prime}<x$. Similarly, if we know that $\pi / 12 n$ is an angular invariant for a triangle with angles $p \pi / n$, then we know that $p s \pi / n$ (more precisely, a representative modulo $2 \pi$ ) is the angular invariant of an equilateral triangle in $\mathbf{H}_{\mathbb{C}}^{2}$ with angles $p \pi / n$. Therefore, it is enough to show that $\pi / 12 n$ is the angular 
invariant of a triangle having angles $p \pi / n$ for all sufficiently large $n$, where $p$ is the smallest not prime dividing $12 n$.

From the above, we conclude further that it suffices to show that there exists a function $q(n)$ such that $p<q(n)$ and

$$
1-3 \cos ^{2}(q(n) \pi / n)+2 \cos (\pi / 12 n) \cos ^{3}(q(n) \pi / n)<0
$$

for all sufficiently large $n$. To prove this, we consider the function $j(n)$, defined in [Jacobsthal 1961]. For any integer $n, j(n)$ is the smallest integer such that any $j(n)$ consecutive integers must contain one that is relatively prime to $n$. Clearly $p \leq j(12 n)$.

Iwaniec [1978] proved that

$$
j(n) \ll(\log n)^{2} .
$$

Therefore, for any $\epsilon>0$, there is an $n_{\epsilon}$ so that the first prime number coprime to $12 n$ is at most $(\log 12 n)^{2+\epsilon}$ for every $n \geq n_{\epsilon}$. Consider the function

$$
f_{\epsilon}(x)=1-3 \cos ^{2}\left(\log (12 / x)^{2+\epsilon} \pi x\right)+2 \cos (\pi x / 12) \cos ^{3}\left(\log (12 / x)^{2+\epsilon} \pi x\right) .
$$

Then $\lim _{x \rightarrow 0} f_{\epsilon}(x)$ exists and equals 0 for all $\epsilon>0$. Further, $x=0$ is a local maximum of $f_{\epsilon}$, so $f_{\epsilon}(1 / n)<0$ for all sufficiently large $n$.

Taking $q(n)=(\log n)^{2+\epsilon}$ for any small $\epsilon$ shows that (8) holds for all sufficiently large $n$. This implies that (7) is negative for all large $n$. This proves the theorem.

Unfortunately, the proof of Theorem 1.7 isn't effective, so we cannot list the angles that can possibly determine an arithmetic lattice. In particular, we don't know which $n$ makes the bound from [Iwaniec 1978] effective for any $\epsilon>0$. If this bound is less than $n=10^{5}$ for some $\epsilon$, which computer experiments show is extraordinarily likely, then we obtain $n<5,000,000$. We expect the actual bound to be quite a bit smaller, especially given that the smallest equilateral triangle in $\mathbf{H}^{2}$ that defines an arithmetic Fuchsian group has angles $\pi / 15$.

\section{Acknowlegments}

I thank the referee for several helpful comments.

\section{References}

[Brehm 1990] U. Brehm, "The shape invariant of triangles and trigonometry in two-point homogeneous spaces", Geom. Dedicata 33:1 (1990), 59-76. MR 91c:53048 Zbl 0695.53038

[Deraux 2006] M. Deraux, "Deforming the $\mathbb{R}$-Fuchsian (4, 4, 4)-triangle group into a lattice", Topology 45:6 (2006), 989-1020. MR 2007m:32015 Zbl 1120.20052

[Deraux et al. 2011] M. Deraux, J. R. Parker, and J. Paupert, "Census of the complex hyperbolic sporadic triangle groups”, Exp. Math. 20:4 (2011), 467-486. MR 2859902 
[Goldman 1999] W. M. Goldman, Complex hyperbolic geometry, Oxford University Press, New York, 1999. MR 2000g:32029 Zbl 0939.32024

[Goldman and Parker 1992] W. M. Goldman and J. R. Parker, "Complex hyperbolic ideal triangle groups”, J. Reine Angew. Math. 425 (1992), 71-86. MR 93c:20076 Zbl 0739.53055

[Holzapfel 1998] R.-P. Holzapfel, Ball and surface arithmetics, Aspects of Mathematics E29, Vieweg, Braunschweig, 1998. MR 2000d:14044 Zbl 0980.14026

[Iwaniec 1978] H. Iwaniec, "On the problem of Jacobsthal”, Demonstratio Math. 11:1 (1978), 225231. MR 80h:10053 Zbl 0378.10029

[Jacobsthal 1961] E. Jacobsthal, "Über Sequenzen ganzer Zahlen, von denen keine zu $n$ teilerfremd ist”, Norske Vid. Selsk. Forh. Trondheim 33 (1961), 117-124. MR 23 \#A2354 Zbl 0096.26002

[Knus et al. 1998] M.-A. Knus, A. Merkurjev, M. Rost, and J.-P. Tignol, The book of involutions, American Mathematical Society Colloquium Publications 44, American Mathematical Society, Providence, RI, 1998. MR 2000a:16031 Zbl 0955.16001

[Mostow 1980] G. D. Mostow, "On a remarkable class of polyhedra in complex hyperbolic space", Pacific J. Math. 86:1 (1980), 171-276. MR 82a:22011 Zbl 0456.22012

[Parker 2008] J. R. Parker, "Unfaithful complex hyperbolic triangle groups, I: Involutions", Pacific J. Math. 238: 1 (2008), 145-169. MR 2009h:20056

[Parker and Paupert 2009] J. R. Parker and J. Paupert, "Unfaithful complex hyperbolic triangle groups, II: Higher order reflections", Pacific J. Math. 239:2 (2009), 357-389. MR 2009h:20057

[Paupert 2010] J. Paupert, "Unfaithful complex hyperbolic triangle groups, III: Arithmeticity and commensurability”, Pacific J. Math. 245:2 (2010), 359-372. MR 2011d:20094

[Platonov and Rapinchuk 1994] V. Platonov and A. Rapinchuk, Algebraic groups and number theory, Pure and Applied Mathematics 139, Academic Press, Boston, MA, 1994. MR 95b:11039 Zbl 0841.20046

[Pratoussevitch 2005] A. Pratoussevitch, "Traces in complex hyperbolic triangle groups", Geom. Dedicata 111 (2005), 159-185. MR 2006d:32036 Zbl 1115.32015

[Raghunathan 1972] M. S. Raghunathan, Discrete subgroups of Lie groups, Ergebnisse der Math. 68, Springer, New York, 1972. MR 58 \#22394a

[Schwartz 2002] R. E. Schwartz, "Complex hyperbolic triangle groups”, pp. 339-349 in Proceedings of the International Congress of Mathematicians (Beijing, 2002), vol. 2, edited by T. T. Li et al., Higher Ed. Press, Beijing, 2002. MR 2004b:57002 Zbl 1022.53034

[Takeuchi 1977] K. Takeuchi, "Arithmetic triangle groups", J. Math. Soc. Japan 29:1 (1977), 91106. MR 55 \#2754 Zbl 0344.20035

[Vinberg 1971] È. B. Vinberg, "Rings of definition of dense subgroups of semisimple linear groups.", Izv. Akad. Nauk SSSR Ser. Mat. 35 (1971), 45-55. MR 43 \#4929

Received October 12, 2011. Revised November 30, 2011.

\section{Matthew Stover}

DEPARTMENT OF MATHEMATICS

UNIVERSITY OF MICHIGAN

530 CHURCH STREET

ANN ARBOR, MI 48109

UNITED STATES

stoverm@umich.edu 


\title{
PACIFIC JOURNAL OF MATHEMATICS
}

\author{
http://pacificmath.org \\ Founded in 1951 by \\ E. F. Beckenbach (1906-1982) and F. Wolf (1904-1989)
}

\section{EDITORS}

V. S. Varadarajan (Managing Editor)

Department of Mathematics

University of California

Los Angeles, CA 90095-1555

pacific@math.ucla.edu

Vyjayanthi Chari

Department of Mathematics

University of California

Riverside, CA 92521-0135

chari@math.ucr.edu

\section{Robert Finn}

Department of Mathematics Stanford University

Stanford, CA 94305-2125

finn@math.stanford.edu

Kefeng Liu

Department of Mathematics

University of California

Los Angeles, CA 90095-1555

liu@math.ucla.edu
Darren Long

Department of Mathematics

University of California

Santa Barbara, CA 93106-3080

long@math.ucsb.edu

Jiang-Hua Lu

Department of Mathematics

The University of Hong Kong

Pokfulam Rd., Hong Kong jhlu@maths.hku.hk

Alexander Merkurjev

Department of Mathematics

University of California

Los Angeles, CA 90095-1555

merkurev@math.ucla.edu
Sorin Popa

Department of Mathematics University of California

Los Angeles, CA 90095-1555 popa@math.ucla.edu

Jie Qing

Department of Mathematics

University of California

Santa Cruz, CA 95064

qing@cats.ucsc.edu

Jonathan Rogawski

Department of Mathematics

University of California

Los Angeles, CA 90095-1555

jonr@math.ucla.edu

\section{PRODUCTION}

pacific@math.berkeley.edu

\section{SUPPORTING INSTITUTIONS}

ACADEMIA SINICA, TAIPEI

CALIFORNIA INST. OF TECHNOLOGY INST. DE MATEMÁTICA PURA E APLICADA KEIO UNIVERSITY

MATH. SCIENCES RESEARCH INSTITUTE NEW MEXICO STATE UNIV.

OREGON STATE UNIV.

\author{
STANFORD UNIVERSITY \\ UNIV. OF BRITISH COLUMBIA \\ UNIV. OF CALIFORNIA, BERKELEY \\ UNIV. OF CALIFORNIA, DAVIS \\ UNIV. OF CALIFORNIA, LOS ANGELES \\ UNIV. OF CALIFORNIA, RIVERSIDE \\ UNIV. OF CALIFORNIA, SAN DIEGO \\ UNIV. OF CALIF., SANTA BARBARA
}

\author{
UNIV. OF CALIF., SANTA CRUZ \\ UNIV. OF MONTANA \\ UNIV. OF OREGON \\ UNIV. OF SOUTHERN CALIFORNIA \\ UNIV. OF UTAH \\ UNIV. OF WASHINGTON \\ WASHINGTON STATE UNIVERSITY
}

These supporting institutions contribute to the cost of publication of this Journal, but they are not owners or publishers and have no responsibility for its contents or policies.

See inside back cover or pacificmath.org for submission instructions.

The subscription price for 2012 is US \$420/year for the electronic version, and \$485/year for print and electronic.

Subscriptions, requests for back issues from the last three years and changes of subscribers address should be sent to Pacific Journal of Mathematics, P.O. Box 4163, Berkeley, CA 94704-0163, U.S.A. Prior back issues are obtainable from Periodicals Service Company, 11 Main Street, Germantown, NY 12526-5635. The Pacific Journal of Mathematics is indexed by Mathematical Reviews, Zentralblatt MATH, PASCAL CNRS Index, Referativnyi Zhurnal, Current Mathematical Publications and the Science Citation Index.

The Pacific Journal of Mathematics (ISSN 0030-8730) at the University of California, c/o Department of Mathematics, 969 Evans Hall, Berkeley, CA 94720-3840, is published monthly except July and August. Periodical rate postage paid at Berkeley, CA 94704, and additional mailing offices. POSTMASTER: send address changes to Pacific Journal of Mathematics, P.O. Box 4163, Berkeley, CA 94704-0163.

PJM peer review and production are managed by EditFLOW ${ }^{\mathrm{TM}}$ from Mathematical Sciences Publishers.

PUBLISHED BY PACIFIC JOURNAL OF MATHEMATICS

at the University of California, Berkeley 94720-3840

A NON-PROFIT CORPORATION

Typeset in LATEX

Copyright $(02012$ by Pacific Journal of Mathematics 


\section{PACIFIC JOURNAL OF MATHEMATICS}

Volume $257 \quad$ No. $1 \quad$ May 2012

Energy and volume of vector fields on spherical domains

FABiano G. B. BRito, ANDRÉ O. Gomes and Giovanni S. Nunes

Maps on 3-manifolds given by surgery

BOLDIZSÁR KALMÁR and ANDRÁS I. STIPSICZ

Strong solutions to the compressible liquid crystal system

Yu-Ming ChU, Xian-Gao LiU and XIAO LIU

Presentations for the higher-dimensional Thompson groups $n V$

Johanna HenNig and FranCESCo MatuCCI

Resonant solutions and turning points in an elliptic problem with oscillatory

boundary conditions

ALFONSO CASTRO and ROSA PARDO

Relative measure homology and continuous bounded cohomology of topological pairs

\section{Roberto Frigerio and CRISTINA PAgLiAnTINi}

Normal enveloping algebras

ALEXANDRE N. GrishKov, Marina RASSKazova and SALVATORE SICILIANO

Bounded and unbounded capillary surfaces in a cusp domain

YASUNORI AOKI and DAVID SIEGEL

On orthogonal polynomials with respect to certain discrete Sobolev inner product

Francisco Marcellán, Ramadan Zejnullahu, Bujar Fejzullahu and EDMUNDO HUERTAS

Green versus Lempert functions: A minimal example

PASCAL THOMAS

Differential Harnack inequalities for nonlinear heat equations with potentials under the Ricci flow

JIA-YONG WU

On overtwisted, right-veering open books

PAOLO LISCA

Weakly Krull domains and the composite numerical semigroup ring $D+E\left[\Gamma^{*}\right]$

JUNG WOOK LIM

Arithmeticity of complex hyperbolic triangle groups 